\title{
Expression of cancer stem cell markers in pancreatic intraepithelial neoplasias and pancreatic ductal adenocarcinomas
}

\author{
SHOKO KURE $^{1}$, YOKO MATSUDA ${ }^{1}$, MASAHITO HAGIO $^{1}$, JUNJI UEDA ${ }^{2}$, \\ ZENYA NAITO $^{1}$ and TOSHIYUKI ISHIWATA ${ }^{1}$ \\ ${ }^{1}$ Departments of Pathology and Integrative Oncological Pathology, Nippon Medical School, and \\ ${ }^{2}$ Surgery for Organ and Biological Regulation, Graduate School of Medicine, Nippon Medical School, Tokyo 113-8602, Japan
}

Received May 2, 2012; Accepted June 28, 2012

DOI: 10.3892/ijo.2012.1565

\begin{abstract}
Cancer stem cells (CSCs) play pivotal roles in cancer growth, invasion, metastasis and recurrence. Several proteins have been reported as CSC markers for pancreatic ductal adenocarcinoma (PDAC). In the present study, we examined the correlation between pancreatic intraepithelial neoplasias (PanINs) and CSC markers including CD24, CD44, CD133, CXCR4, ESA and nestin using immunohistochemical analysis. Furthermore, we examined the roles and clinical significance of these CSC markers in PDAC. CD24-, CD44-, CXCR4-, ESA- and nestin-positive cells were detected in the following tissues, listed in order of increasing percentage: normal ducts $<$ low-grade PanINs < high-grade PanINs < PDACs. CD133 did not increase according to the malignancy grade. In PDAC, cells positive for each of the following CSC markers were detected, listed according to increasing percentage: nestin $<\mathrm{CD} 133<\mathrm{CD} 44<\mathrm{CD} 24<\mathrm{CXCR} 4<\mathrm{ESA}$. CXCR4 and ESA expression correlated with well-differentiated PDAC. Venous invasion was positively associated with CD133 and inversely associated with ESA. CSC marker expression levels detected in PDAC cell lines using flow cytometry showed lowest expression of CD133 and highest of CD44, differing from the results obtained using immunohistochemistry. In two PDAC subtypes, adenosquamous carcinoma and anaplastic carcinoma, ESA was expressed more abundantly in adenocarcinoma components, whereas CD44 and nestin showed high expression in anaplastic components. Together, these results suggest that most CSC markers correlate with pancreatic carcinogenesis through the PanIN-to-PDAC sequence. Each CSC marker was related in a different manner with proliferation, differentiation, invasiveness or tissue type of PDAC.
\end{abstract}

Correspondence to: Dr Toshiyuki Ishiwata, Departments of Pathology and Integrative Oncological Pathology, Nippon Medical School, 1-1-5 Sendagi, Bunkyo-ku, Tokyo 113-8602, Japan

E-mail: ishiwata@nms.ac.jp

Key words: cancer stem cell, pancreatic intraepithelial neoplasia, pancreatic ductal adenocarcinoma, immunohistochemistry

\section{Introduction}

Cancer cells that survive after treatment can cause local recurrence and distant metastases. A hierarchical model proposes that tumor consists of proliferative cells, which have limited life spans, and CSCs, which can self-renew, undergo multilineage differentiation, and are suited to survive adverse conditions in the tissue microenvironment (1). This cancer stem cell (CSC) hypothesis may account for carcinogenesis, the functional heterogeneity observed in tumor tissues, and tumor relapse after treatment due to the presence of a therapyresistant population (2).

Pancreatic ductal adenocarcinoma (PDAC) has a high mortality rate due to its aggressive growth and high metastatic rates (3). Multiple lines of evidence have shown the existence of CSC fractions in PDAC (4), which have been detected by CSC-specific markers, including CD24 (5-7), CD44 (5,6,8,9), CD133 (10-13), CXCR4 (10,14), epithelial cell adhesion molecule (EpCAM; epithelial-specific antigen, ESA) (5), nestin (15), and combinations of these markers $(5,10)$. However, definitive CSC markers for PDAC and their clinical relevance are still controversial (16).

There is substantial evidence that PDAC does not arise de novo, but rather progresses through a multistep model involving non-invasive precursor lesions known as pancreatic intraepithelial neoplasias (PanINs), and culminating in invasive cancer (17). CXCR4 expression begins in PanIN lesions, and the CXCR4-expressing cells appeared to mediate pancreatic cancer metastasis (10). It has also been shown that endogenous K-ras expression in the pancreatic nestin-positive cell lineage induces mouse PanINs (18). These findings led to the hypothesis that CXCR4- or nestin-positive pancreatic cells are cancer-initiating cells of PDAC, which has been considered a property of CSCs. However, the relationship between CSCs and PanIN lesions remains unclear.

Most research related to CSCs has been conducted using established cell lines or experimental animals; thus, little is known about the localization and roles of CSCs in human tissues. In this study, we analyzed the expression levels of CSC markers in different grades of PanINs, using human pancreatic tissues. We also examined the expression and roles of the CSC markers in PDAC tissues, and compared the expression levels of these CSC markers to those of established PDAC cell lines. 
Table I. Normal, PanIN and PDAC cases.

$\mathrm{N}$

\begin{tabular}{lc}
\hline Normal pancreatic tissue & 6 cases $(53$ ducts) \\
PanIN & 41 cases \\
PanIN-1 & 32 ducts \\
PanIN-2 & 50 ducts \\
PanIN-3 & 18 ducts \\
Conventional type of PDAC & 105 cases \\
Adenosquamous carcinoma & 7 cases \\
Anaplastic carcinoma & 1 case
\end{tabular}

\section{Materials and methods}

Materials. The following were purchased: Histofine Simple Stain MAX PO (R) and (M) kits from Nichirei (Tokyo, Japan); mouse monoclonal anti-CD24 antibody from Santa Cruz Biotechnology (Santa Cruz, CA, USA); mouse monoclonal anti-CD44, anti-nestin antibody, mouse IgG1, and mouse IgG2A from R\&D Systems Inc. (Westerville, $\mathrm{OH}$, USA); mouse polyclonal ESA antibody from Abnova (Taipei City, Taiwan), rabbit polyclonal anti-CD133, anti-CXCR4 antibody, rabbit $\mathrm{IgG}$, and mouse $\mathrm{IgG}$ from Abcam plc. (Cambridge, UK); mouse monoclonal anti-Ki 67 antibody and anti-PCNA antibody from Dako Corp. (Santa Barbara, CA, USA); High Capacity cDNA Reverse Transcription kit and TaqMan Gene Expression assay for CD24 (Hs02379687_s1), CD44 (Hs00153304_m1), ESA (Hs00901885_m1), CD133 (Hs01009238_m1), CXCR4 (Hs00607978_s1), nestin (Hs00707120_s1), and 18S ribosomal RNA (18S rRNA, Hs03928990_g1) from Applied Biosystems (Foster City, CA, USA); NucleoSpin RNA II from Takara Biotechnology (Tokyo, Japan); human serum from Lonza (Walkersville, MD, USA); Zenon Labeling kit from Invitrogen Corp. (Carlsbad, CA, USA); and SureLink Fluorescein (FITC) Labeling kit from KPL, Inc. (Gaithersburg, MD, USA). All other chemicals and reagents were purchased from Sigma Chemical Corp. (St. Louis, MO, USA).
Patients and tissues. Tissues from 105 patients with conventional PDAC were obtained for this study (Table I). These patients received treatment at Nippon Medical School Hospital (Tokyo, Japan) between 1995 and 2011. None of the patients received preoperative chemotherapy and radiotherapy. The patients consisted of 61 males and 44 females, with a median age of 68 years (range, 35-87 years). Clinicopathological stages were determined according to the TNM classification system of the Union for International Cancer Control (UICC). The mean follow-up period was 17.2 months (range, 0.4-153.1 months). Forty-six patients did not receive any postoperative chemotherapy, whereas 59 patients received adjuvant chemotherapy following surgery; 34 patients received gemcitabine, 14 received uracil/tegafur, 6 received gemcitabine and tegafur/gimeracil/oteracil potassium, 3 received uracil/ tegafur and gemcitabine and 2 received fluorouracil.

Two PDAC subtypes, adenosquamous carcinomas $(\mathrm{N}=7)$ and anaplastic carcinoma $(\mathrm{N}=1)$, were separately analyzed. The normal pancreatic tissues $(\mathrm{N}=6)$ were obtained from patients who underwent surgery for ectopic spleen. PanIN tissues $(\mathrm{N}=41)$ were obtained from both PDAC and normal tissues. This study was carried out in accordance with the principles embodied in the Declaration of Helsinki, 2008; each patient gave informed consent for the use of the pancreatic tissues.

Immunohistochemistry. Paraffin-embedded sections $(3.5 \mu \mathrm{m})$ were subjected to immunohistochemistry (IHC). After deparaffinization, antigen retrieval was performed (except for nestin) at $121^{\circ} \mathrm{C}$ for $15 \mathrm{~min}$ in a sodium citrate buffer solution (pH 6.0). Endogenous peroxidase activity was blocked by incubation for $30 \mathrm{~min}$ with $0.3 \%$ hydrogen peroxide in methanol. The tissue sections were then incubated with each antibody in phosphate-buffered saline (PBS) containing $1 \%$ bovine serum albumin (BSA) overnight at $4^{\circ} \mathrm{C}$. The dilutions of each primary antibody are listed in Table II. Bound antibodies were detected with Simple Stain MAX PO (R) or $(\mathrm{M})$ reagent, using diaminobenzidine tetrahydrochloride as the substrate. The sections were then counterstained with Mayer's hematoxylin. Negative control tissue sections were prepared by omitting the primary antibody.

Table II. Antibodies and their application for immunohistochemistry and flow cytometry.

\begin{tabular}{lll}
\hline & & \multicolumn{1}{c}{ Dilution } \\
\cline { 2 - 3 } Primary antibodies & Isotype control & IHC \\
\hline CD24, Santa Cruz Biotechnology, SC-19585 & mouse IgG1 & $1: 100$ \\
CD44, R\&D Systems, BBA10 & mouse IgG2A & $1: 1000$ \\
CD133, Abcam, ab19898 & rabbit IgG & $1: 800$ \\
CXCR4, Abcam, ab2074 & rabbit IgG & $1: 200$ \\
ESA, Abnova, H4072-B02P & mouse IgG & $1: 100$ \\
Nestin, R\&D Systems, MAB1259 & mouse IgG1 & $1: 500$ \\
\hline
\end{tabular}

IHC, immunohistochemistry; FCM, flow cytometry. 


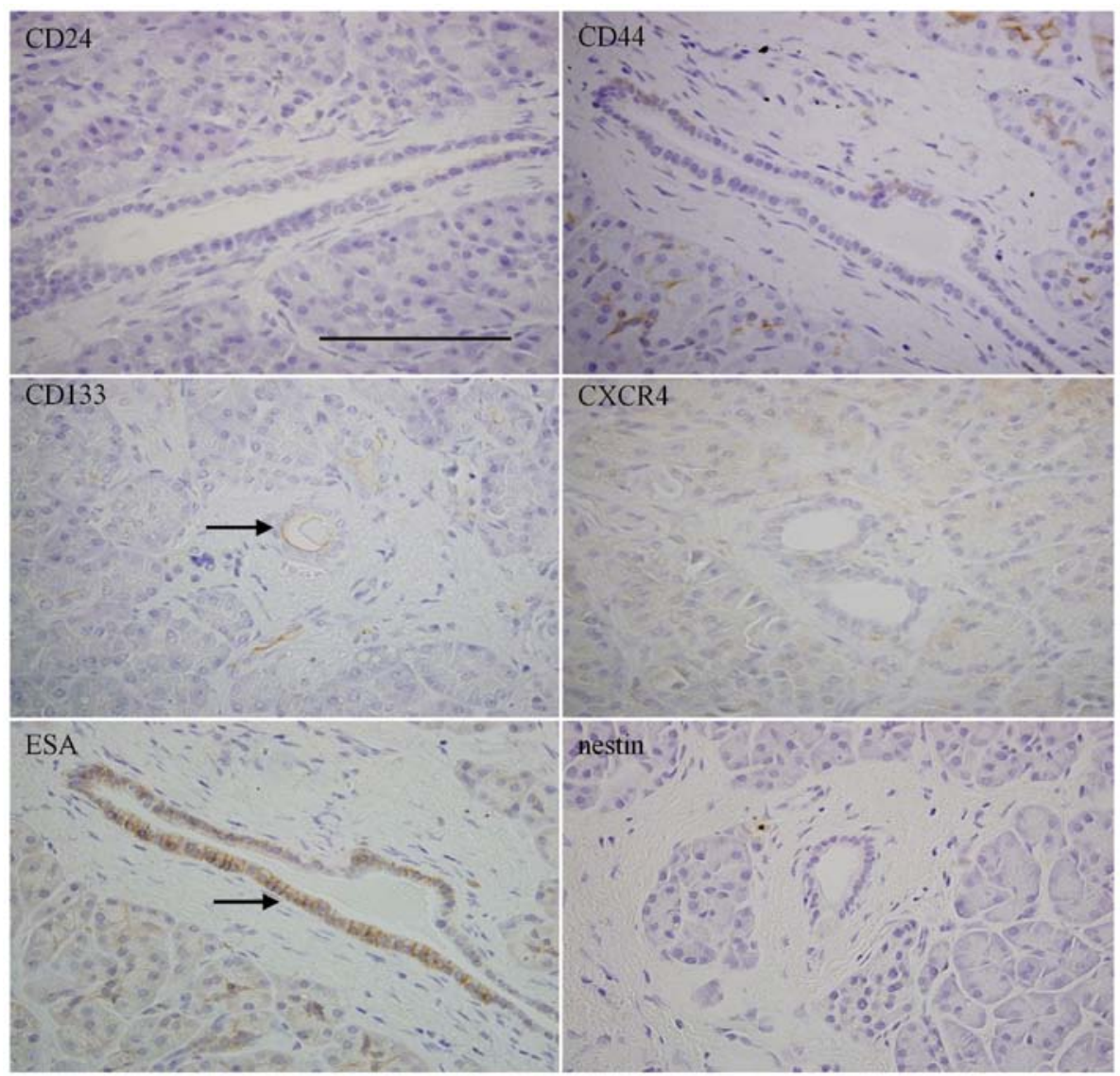

Figure 1. Immunohistochemical staining of each CSC marker in normal pancreatic tissues. The arrows indicate positive staining of CD133 and ESA. Bar, $100 \mu \mathrm{m}$.

IHC staining was evaluated independently by three investigators (S.K. and Y.M. for PanIN and Y.M and T.I. for PDAC) who were blind to clinical and outcome data. Percentages of positive cells $(0-100 \%)$ were evaluated for each stained sample. For PDACs, evaluation was performed within the tumor area of an individual slide. For PanINs and normal ducts, one or more ducts were evaluated from one slide (10) and each case or duct was subdivided into high- and low-expression groups according to the median percentage of positive cells for each marker.

PDAC cell lines. KLM-1, PANC-1, MIA PaCa-2, PK-1, PK-45H and PK-8 PDAC cell lines were obtained from the Cell Resource Center for Biomedical Research, Institute of Development, Aging and Cancer, Tohoku University (Sendai, Japan), and Capan-1 was purchased from the American Type Culture Collection. The AsPC-1 cell line was obtained from Dainippon Sumitomo Pharma Co. Ltd. (Osaka, Japan). The cells were grown in RPMI-1640 medium containing 10\% fetal bovine serum (FBS) at $37^{\circ} \mathrm{C}$ under a humidified $5 \% \mathrm{CO}_{2}$ atmosphere. Capan-1 cells were incubated in the same medium with $15 \%$ FBS.

Quantitative real-time PCR of CSC markers in pancreatic cancer cells. All PDAC cells were seeded at $2.5 \times 10^{5}$ cells per $60-\mathrm{mm}$ dish, and were incubated for $48 \mathrm{~h}$. Total RNA extraction was performed using NucleoSpin RNA II. Then, cDNA synthesis was performed using a High Capacity cDNA Reverse
Transcription kit, following the manufacturer's protocol. Quantitative real-time PCR (qRT-PCR) was performed using the StepOnePlus Real-time PCR systems (Applied Biosystems, Inc.). The PCR reaction mixture $(18 \mu \mathrm{l})$, containing $2 \mu \mathrm{l}$ of template cDNA, $10 \mu \mathrm{l}$ of TaqMan Fast Universal PCR Master Mix, and $1 \mu \mathrm{l}$ of the TaqMan Gene Expression assay for CD24, CD44, CD133, CXCR4, ESA, or nestin, was placed in a 96-well reaction plate. $18 \mathrm{~S}$ rRNA, as the internal positive control, was amplified using the TaqMan gene expression assay. The optimized program involved denaturation at $95^{\circ} \mathrm{C}$ for $20 \mathrm{sec}$, followed by 40 cycles of amplification $\left(95^{\circ} \mathrm{C}\right.$ for $1 \mathrm{sec}$ and $60^{\circ} \mathrm{C}$ for $20 \mathrm{sec}$ ). Results were expressed as target/18S rRNA, as an internal standard concentration ratio. Gene expression measurements were performed in triplicate.

Flow cytometry. For flow cytometry (FCM), we used the same antibodies as were used for IHC (Table II). CD24, CD44, CD133, CXCR4, and nestin antibodies were labeled with allophycocyanin (APC) using the Zenon mouse or rabbit IgG Labeling kit, and ESA antibody was labeled with FITC using the SureLink Labeling kit according to the manufacturer's protocol. Cells were incubated for $20 \mathrm{~min}$ at $4^{\circ} \mathrm{C}$ in PBS containing $10 \%$ human serum. Cells $\left(5 \times 10^{5}\right.$ cells) were then centrifuged briefly to remove the serum-containing medium, and incubated with antibody for $60 \mathrm{~min}$ in the dark at $4^{\circ} \mathrm{C}$; $1 \mu \mathrm{g}$ of propidium iodide was added to label dead cells. To stain nestin, cells were fixed with $4 \%$ paraformaldehyde, and incubated with the anti-nestin antibody and $0.1 \%$ Triton-X for 


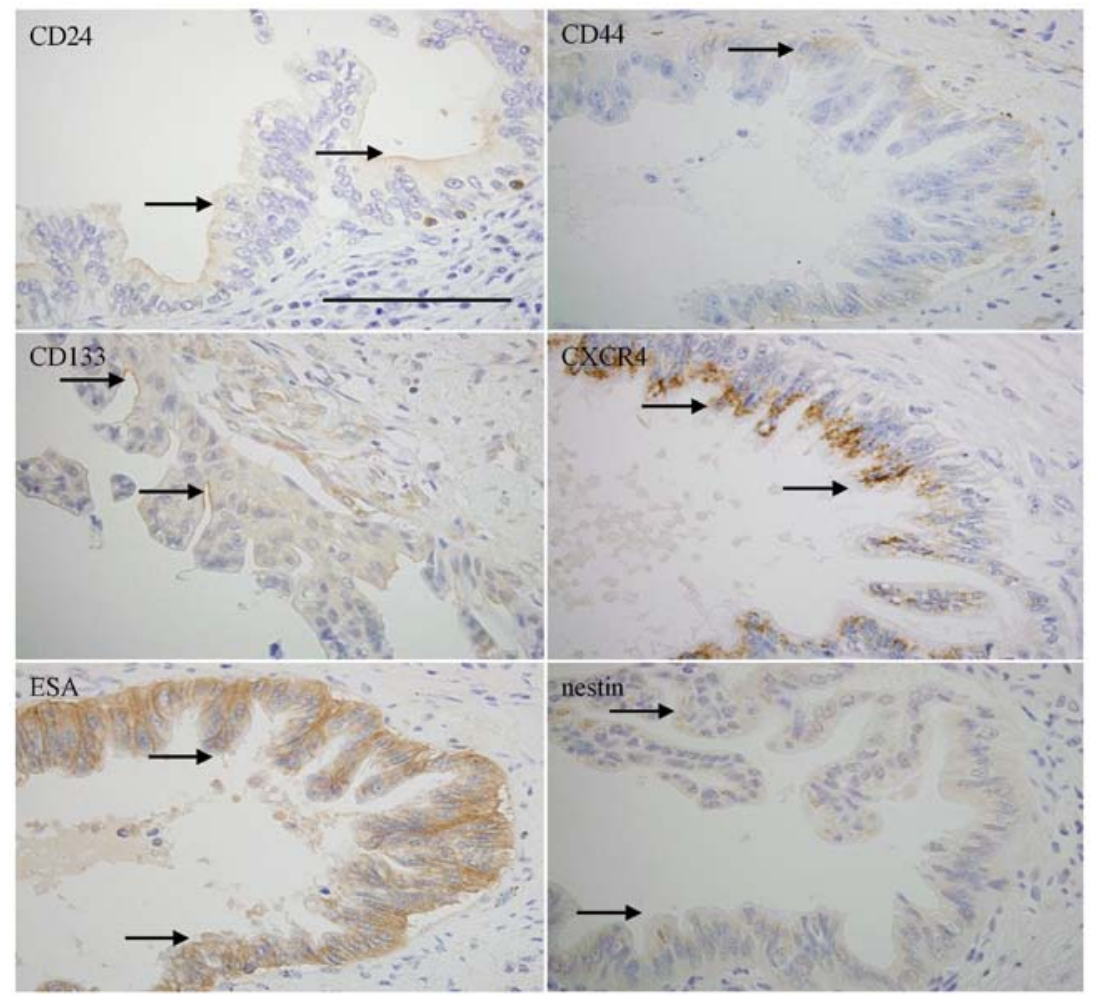

Figure 2. Immunohistochemical staining of each CSC marker in PanIN-3 lesions. The arrows indicate positive staining of CD24, CD44, CD133, CXCR4, ESA, and nestin. Bar, $100 \mu \mathrm{m}$.

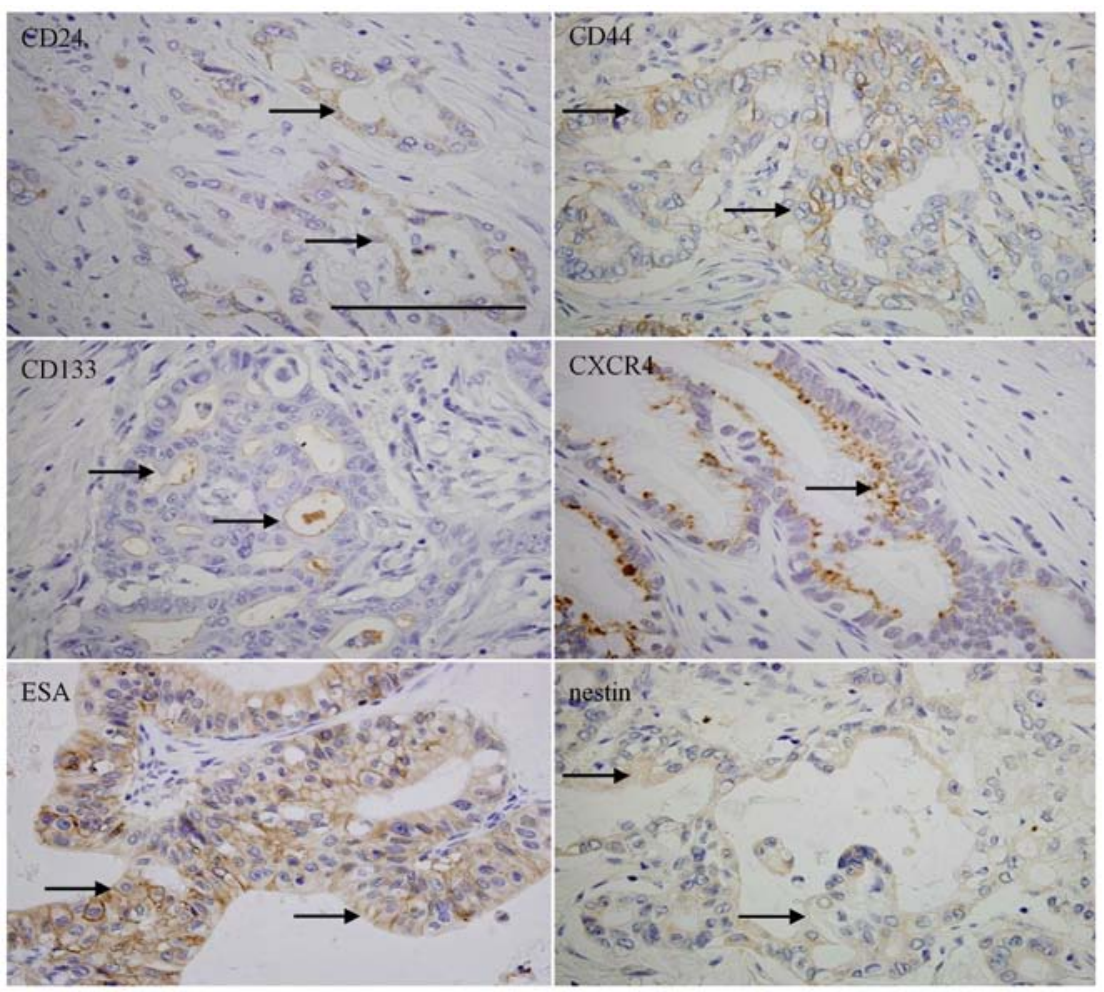

Figure 3. Immunohistochemical staining of each CSC marker in PDACs. The arrows indicate positive staining of CD24, CD44, CD133, CXCR4, ESA and nestin. Bar, $100 \mu \mathrm{m}$.

$60 \mathrm{~min}$ in the dark at room temperature. We prepared isotype control-treated cells as a negative control (Table II). Each marker's expression was determined using a BD FACSAria II flow cytometer (BD Bioscience, Franklin Lakes, NJ, USA) and analyzed using FlowJo version 7.6.4 (Tree Star, Inc., Ashland, OR, USA). 

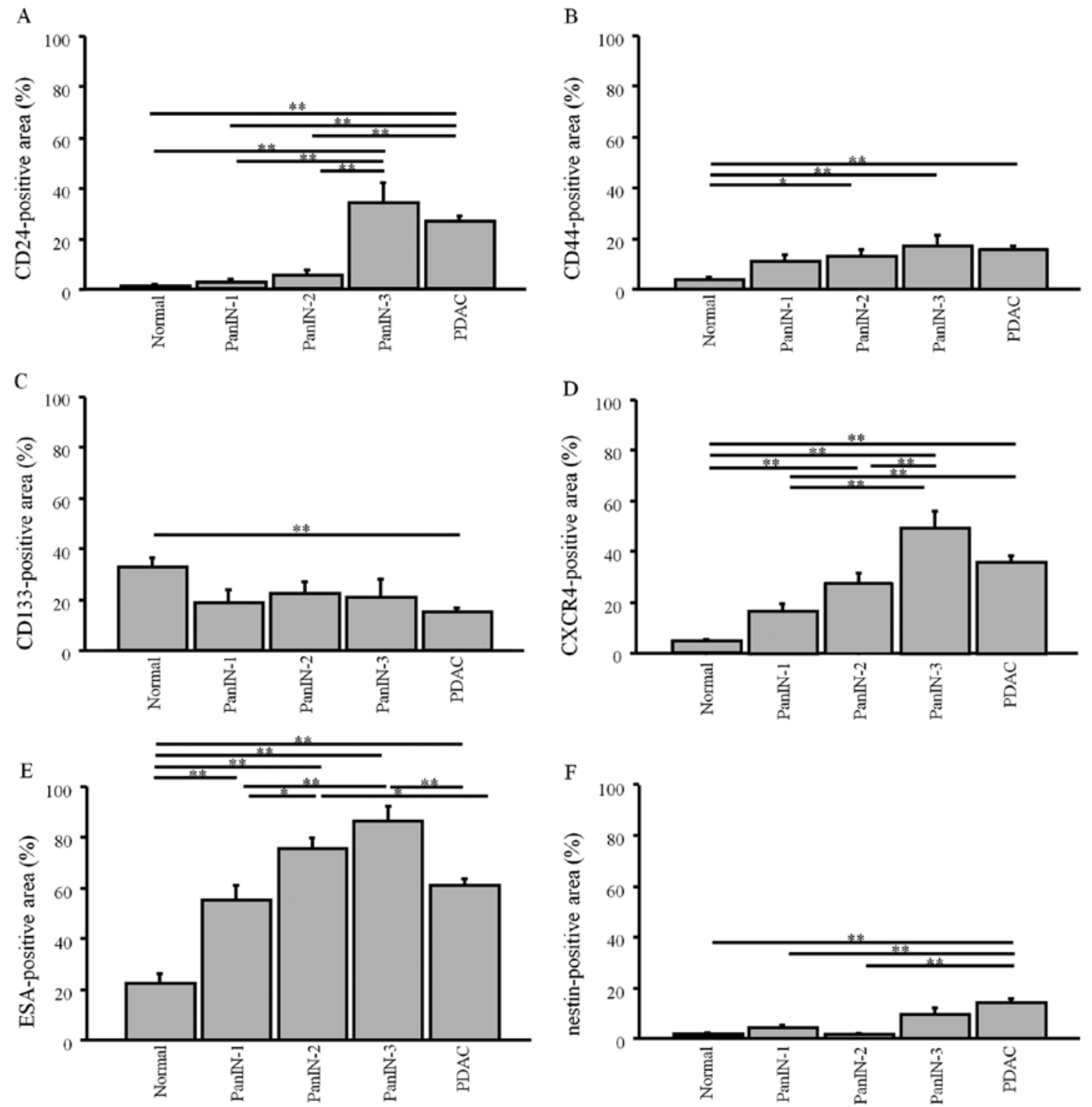

Figure 4. The percentages of cells that were positive for each CSC marker in normal pancreata, PanINs, and PDACs. Normal, N=53 ducts; PanIN-1, N=32 ducts; PanIN-2, N=50 ducts; PanIN-3, N=18 ducts; PDAC, N=105 cases. (A) CD24; (B) CD44; (C) CD133; (D) CXCR4; (E) ESA; (F) nestin. "P<0.05; ** P<0.01.

Statistical analysis. Results for cell proliferation are shown as mean $\pm \mathrm{SE}$, and the data between different groups were compared using the Student's t-test. Whenever indicated, the Chi-square test and Fisher's exact test were used to analyze the correlation between marker expression and clinicopathological features. Cumulative survival rates were calculated by the Kaplan-Meier method, and the significance of differences in survival rate was analyzed by the log-rank test. Data between multiple groups were compared using one-way ANOVA, then were analyzed using a post-hoc test. $\mathrm{P}<0.05$ was considered significant in all analyses. Computations were performed using the StatView J version 5.0 software package (SAS Institute, Inc., Cary, NC, USA).

\section{Results}

Immunohistochemistry of CSCs markers in normal, PanINs and PDAC. First, we conducted immunohistochemical analyses of various CSC markers, in order to confirm the localizations and differences among normal, PanIN, and
PDAC tissues. Normal pancreatic tissues were mostly negative for CD24, CD44, CXCR4, and nestin, whereas CD133 and ESA were relatively highly expressed in ductal cells (Fig. 1). PanIN-3 lesions showed weakly positive expressions of CD44, CD133, and nestin in ductal cells (Fig. 2). CD24, CXCR4 and ESA were moderately to strongly expressed in the ductal cells of PanIN-3 lesions, with different intracellular localization patterns: CD24 in luminal surface of cell membrane, CXCR4 in cells with a granular staining pattern, and ESA diffusely distributed both in cell membrane and cytoplasm (Fig. 2). PDAC showed expressions of CD24, CD44, and CD133 in cell membranes of cancer cells; CXCR4 and nestin were expressed in cytoplasm, and ESA was expressed in both cytoplasm and cell membrane (Fig. 3).

Next, we compared the percentages of immunohistochemical expression of each CSC marker in normal, PanIN, and PDAC tissues (Table I; Fig. 4). The expression levels of CD24, CD44, CXCR4, ESA, and nestin showed tendencies to increase according to the malignancy grade of PanINs, but CD133 showed an opposite trend (Fig. 4). As compared 
Table III. Expression of each CSC marker in IHC and FCM.

\begin{tabular}{|c|c|c|c|c|c|c|}
\hline & $\mathrm{CD} 24$ & CD44 & CD133 & CXCR4 & ESA & Nestin \\
\hline \multicolumn{7}{|l|}{ IHC } \\
\hline Normal & $0.6 \pm 0.6$ & $3.0 \pm 1.2$ & $32.3 \pm 3.9$ & $4.2 \pm 1.1$ & $21.7 \pm 3.7$ & $0.1 \pm 0.1$ \\
\hline PanIN-1 & $2.0 \pm 1.2$ & $10.5 \pm 2.7$ & $18.5 \pm 4.9$ & $15.9 \pm 3.5$ & $54.9 \pm 5.6$ & $3.4 \pm 1.3$ \\
\hline PanIN-2 & $4.4 \pm 2.0$ & $12.2 \pm 2.4$ & $21.6 \pm 4.5$ & $26.7 \pm 4.1$ & $75.1 \pm 3.9$ & $1.0 \pm 0.3$ \\
\hline PanIN-3 & $33.1 \pm 8.0$ & $16.7 \pm 4.3$ & $20.3 \pm 7.3$ & $48.9 \pm 6.7$ & $86.1 \pm 5.8$ & $8.6 \pm 3.1$ \\
\hline PDAC & $26 \pm 2.1$ & $15.1 \pm 1.5$ & $14.5 \pm 1.6$ & $35.6 \pm 2.3$ & $60.6 \pm 2.6$ & $13.5 \pm 1.7$ \\
\hline \multicolumn{7}{|l|}{ FCM } \\
\hline PDAC & $20.6 \pm 13.2$ & $77.4 \pm 16.2$ & $0.54 \pm 0.54$ & $23.8 \pm 11.9$ & $35.1 \pm 29.4$ & $4.4 \pm 3.5$ \\
\hline
\end{tabular}

Data represent mean percentage of each CSC marker \pm SE.
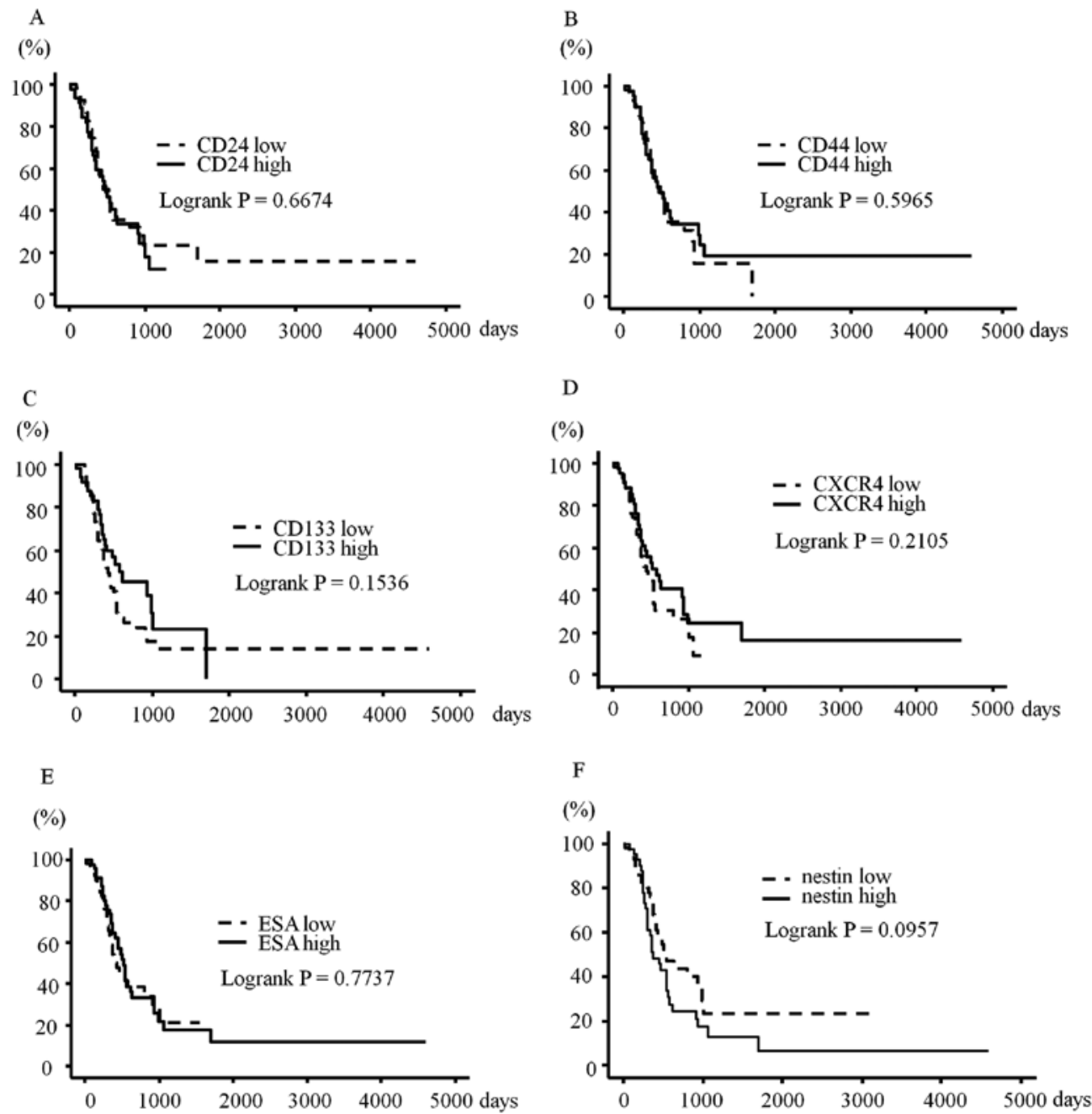

Figure 5. Kaplan-Meier curves for overall survival according to high- and low-expression groups for each CSC marker. The mean survival time was 17.2 months (range, 0.4-153.1 months). N=105 cases. (A) CD24; (B) CD44; (C) CD133; (D) CXCR4; (E) ESA; (F) nestin.

with normal, PanIN-1, and PanIN-2 tissues, significantly increased CD24 expression was observed in PanIN-3 and PDAC (Fig. 4A; ${ }^{* *} \mathrm{P}<0.01$ ). Expressions of CD44 and CXCR4 were significantly increased in PanIN-2, PanIN-3 and PDAC (Fig. 4B and $\mathrm{D} ;{ }^{*} \mathrm{P}<0.05 ;{ }^{* *} \mathrm{P}<0.01$ ). ESA staining was relatively stronger compared to the other markers, and all grades of
PanINs and PDAC exhibited significantly higher positivity than normal tissues (Fig. 4E; ${ }^{\mathrm{P}}<0.05 ;{ }^{* *} \mathrm{P}<0.01$ ). PDAC showed significantly higher positivity for nestin compared to normal, PanIN-1 and PanIN-2 tissues (Fig. 4F; ${ }^{* *} \mathrm{P}<0.01$ ). In contrast to the other markers, $\mathrm{CD} 133$ showed highest positivity in normal epithelial duct (Fig. 4C; ${ }^{* *} \mathrm{P}<0.01$ ). 

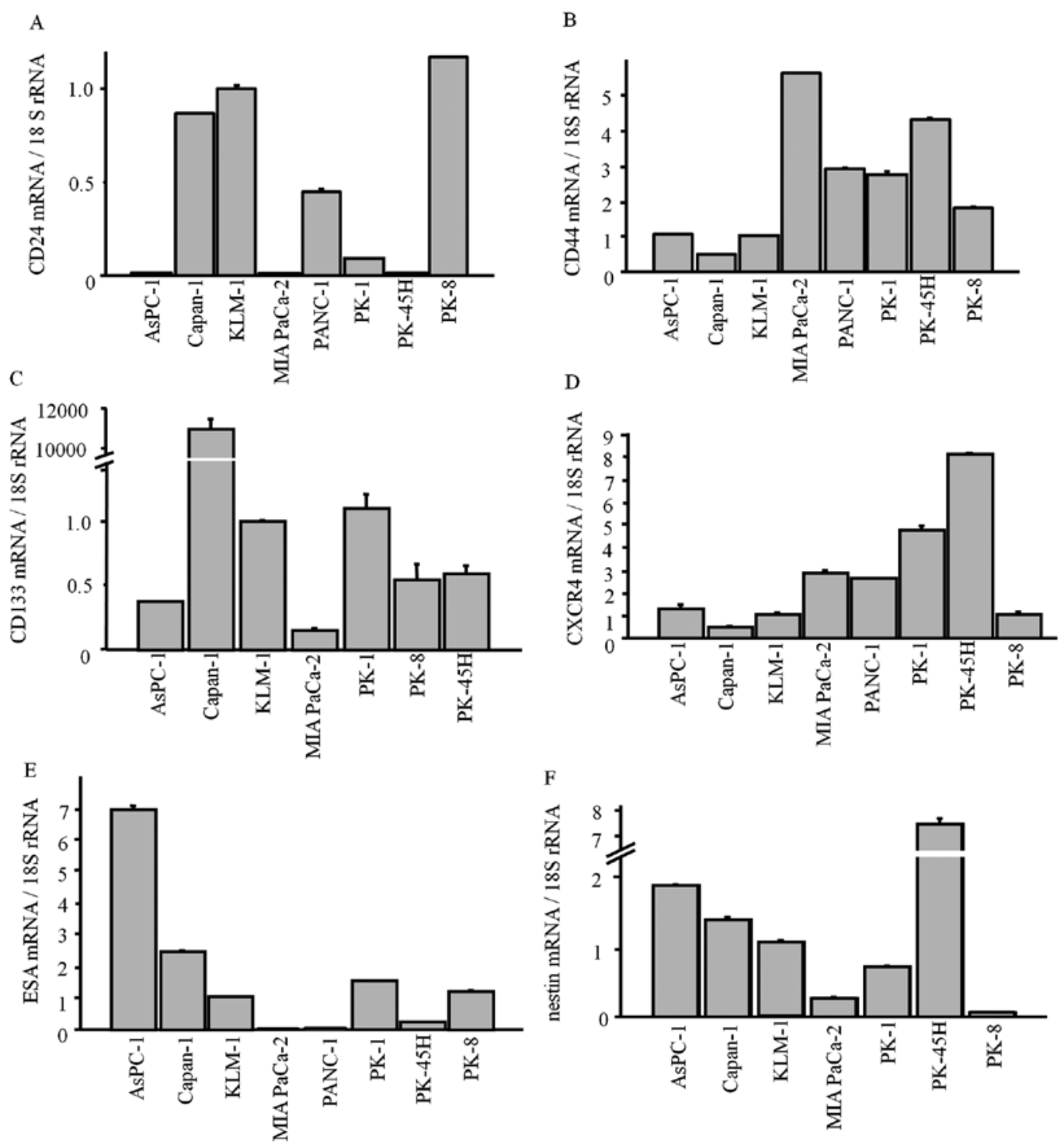

Figure 6. Quantitative real-time PCR analysis of CSC markers in human PDAC cell lines. Data represent mean \pm SE. Y-axis indicates the expression level of mRNA of CSC markers. (A) CD24; (B) CD44; (C) CD133; (D) CXCR4; (E) ESA; (F) nestin.

To confirm that the PanIN lesions selected in the present study possessed high proliferative ability and that they exist as pre-cancerous lesions for PDAC, we analyzed $\mathrm{Ki}$ 67- and PCNA-labeling indices. Both Ki 67- and PCNA-labeling indices increased along with the PanIN grades, and PDAC showed significantly higher proliferative activity than normal and PanIN tissues (data not shown). These findings suggest that CSC markers, including CD24, CD44, CXCR4, ESA and nestin, are involved in proliferative activity or carcinogenesis stages of PDAC via PanIN lesions.

CSC markers and clinicopathological features in PDAC. Next, we examined the correlations between each CSC marker and the clinicopathological features of PDACs. Nestin exhibited the lowest expression, followed, in order of increasing expression, by CD133, CD44, CD24, CXCR4 and ESA (Table III). The CSC marker expression levels widely varied in PDAC tissues; thus, we analyzed clinicopathological aspects for the high- and low-expression groups, using each marker's median as a cut-off. Correlations between the expression levels of each CSC marker and the clinicopathological characteristics of conventional PDAC were analyzed. A statistically significant correlation was found between CD133 expression levels in PDAC and pT stages $(\mathrm{P}=0.0494)$, but these data may be biased by great differences in the number of cases for each T stage. As not expected, ESA and CXCR4 expressions in PDAC were inversely associated with histological grade, with well-differentiated PDAC tending to express CXCR4 and ESA ( $\mathrm{P}=0.0112$ and $\mathrm{P}=0.0058$, respectively). Regarding metastatic features, more severe status of venous invasion was associated with higher $\mathrm{CD} 133$ expression $(\mathrm{P}=0.0056)$ and lower ESA expression $(\mathrm{P}=0.0243)$. There were no significant differences between expression of CSC markers and gender, age and TNM stage. These results may indicate that the expressions of some CSC markers are associated in a different manner with differentiation and invasiveness of PDAC.

CSC markers and prognosis of PDAC cases. Overall survival was not significantly correlated with any of the six CSC markers (Fig. 5). Disease-free survival was not correlated 
PANC-1

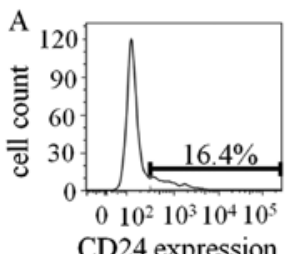

CD24 expression

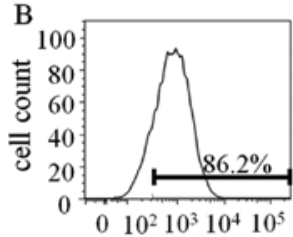

CD44 expression

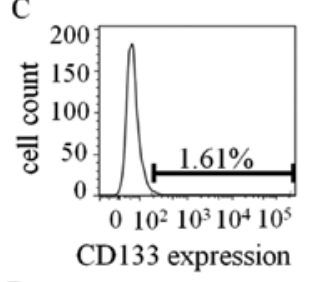

D
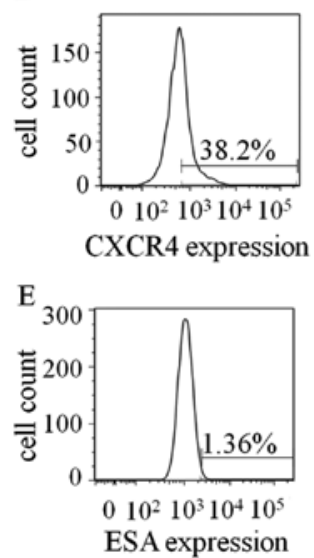

$\mathrm{F}$

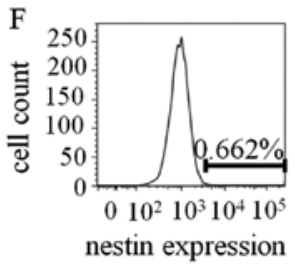

MIA PaCa-2
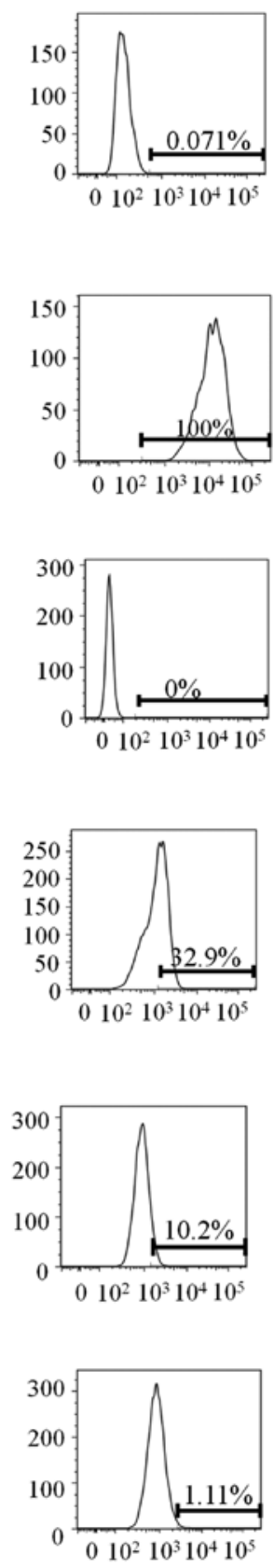

KLM-1
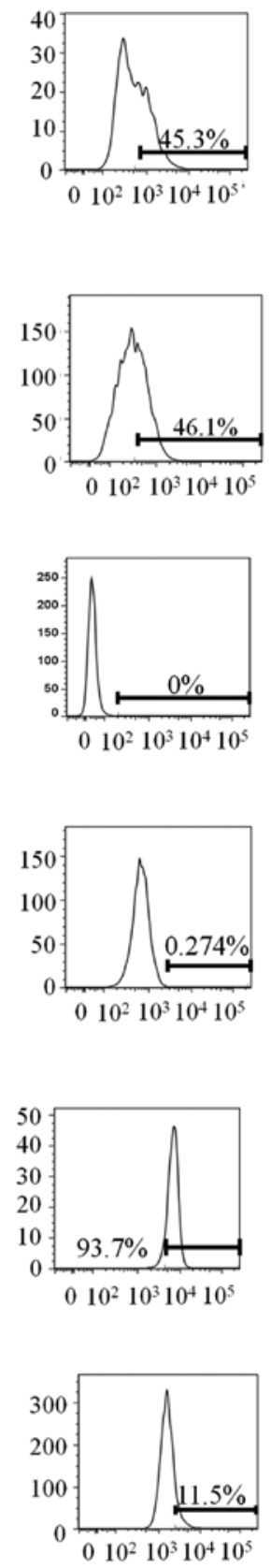

Figure 7. Flow cytometric analysis of CSC markers in human pancreatic cancer cell lines. Bars indicate positive staining fraction for each marker as compared with IgG-matched isotype controls. The number in the graph represents the percentage of positive cells. X-and Y-axis indicate immunofluorescent intensity and the cell count, respectively. (A) CD24; (B) CD44; (C) CD133; (D) CXCR4; (E) ESA; (F) nestin.

with expression of CSC markers (data not shown). However, higher nestin expression tended to be associated with worse overall and disease-free survivals $(\mathrm{P}=0.0957$ and $\mathrm{P}=0.0840$, respectively). Previous reports have shown that analysis of co-expressions of some CSC markers can be more effective for detecting the CSC fraction $(5,10)$; therefore, we also performed survival analysis using co-expressions of CD24, CD44, and ESA, or co-expression of CXCR4 and CD133. These analyses did not find any significant correlations with survival (data not shown). Furthermore, we performed the survival analyses with the cut-off for positivity set at 10 and $30 \%$, but still found no statistically significant correlation (data not shown).
CSC markers in PDAC cell lines. Next, we examined the mRNA levels of each CSC marker, and the corresponding protein levels in established PDAC cell lines. All CSC markers were expressed in the PDAC cell lines at various levels (Fig. 6). As in the immunohistochemistry results, CD133 was weakly expressed in all lines except for Capan-1 (Fig. 6C).

We also conducted FCM using PDAC cell lines, PANC-1, MIA PaCa-2 and KLM-1 (Fig. 7). Expression levels of each CSC marker varied depending on the cell line. Protein levels of CD24, CD44, CXCR4, and nestin were measured by FCM in PANC-1, MIA PaCa-2, and KLM-1 cells and were correlated with their mRNA levels. Consistent with IHC and qRT-PCR 


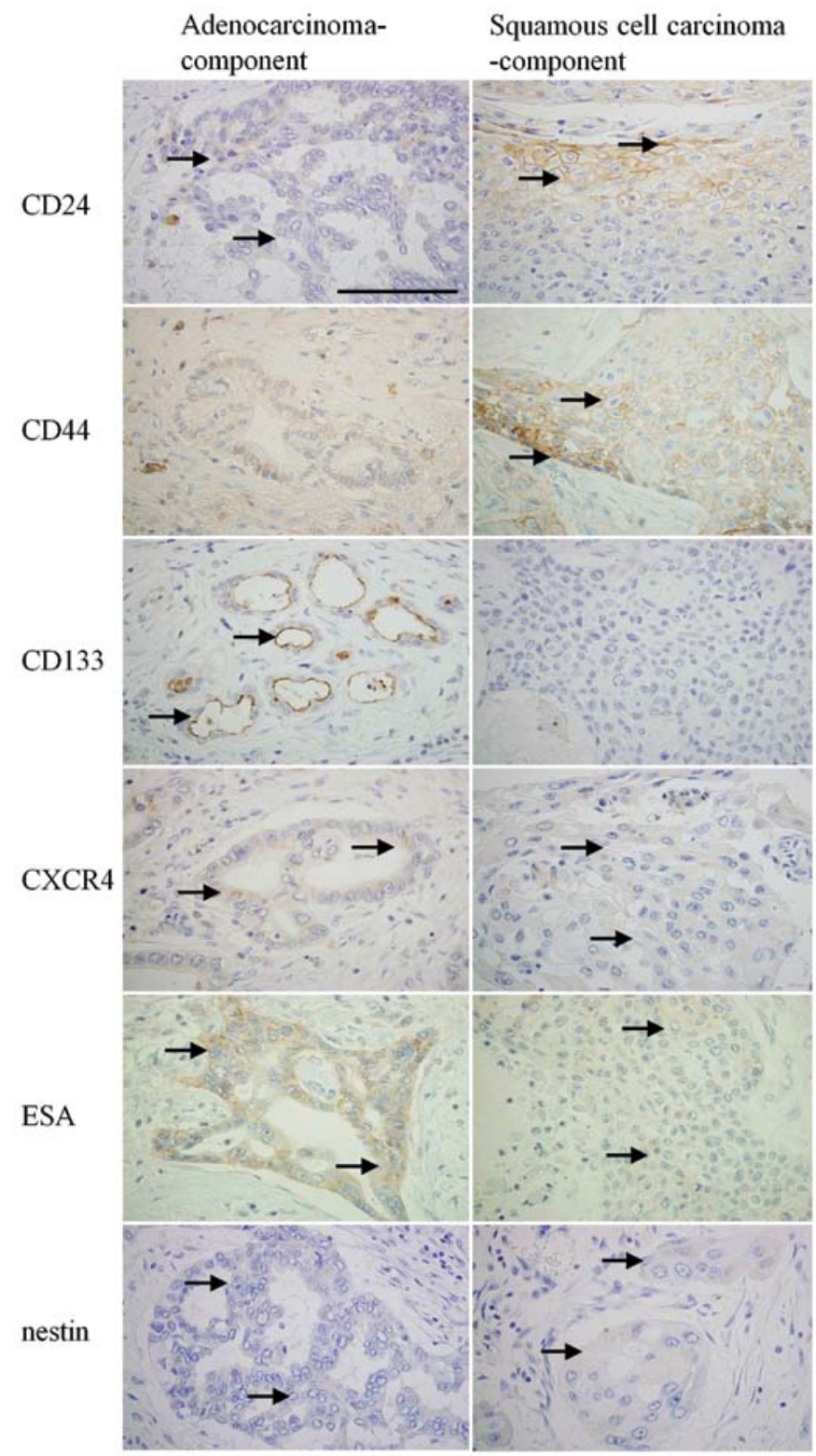

Figure 8. Immunohistochemical staining of CSC markers in adenosquamous carcinomas. Representative adenocarcinoma components and squamous cell carcinoma components in adenosquamous carcinoma are shown in the left and the right panels, respectively. The arrows indicate positive stained areas. Bar, $100 \mu \mathrm{m}$.

analysis, FCM showed very low CD133 expression (Fig. 7C). On the other hand, we observed relatively high expression levels of CD44 and nestin compared to other markers, which differed from the IHC results (Fig. 7B and F). The expression levels of each marker were detected in the following order of increasing percentage in PDAC cell lines: CD133 < nestin $<$ CD24 < CXCR4 < ESA < CD44 (Table III). The order of increasing percentages of CSC markers in PDAC cell lines and PDAC tissues were not identical.

Immunohistochemistry of PDAC subtypes. Each CSC marker showed a tendency of having a different expression pattern depending on histological types or differentiation of PDAC cells, and expressions widely varied between each case and cell line. These findings led us to hypothesize that histolog-

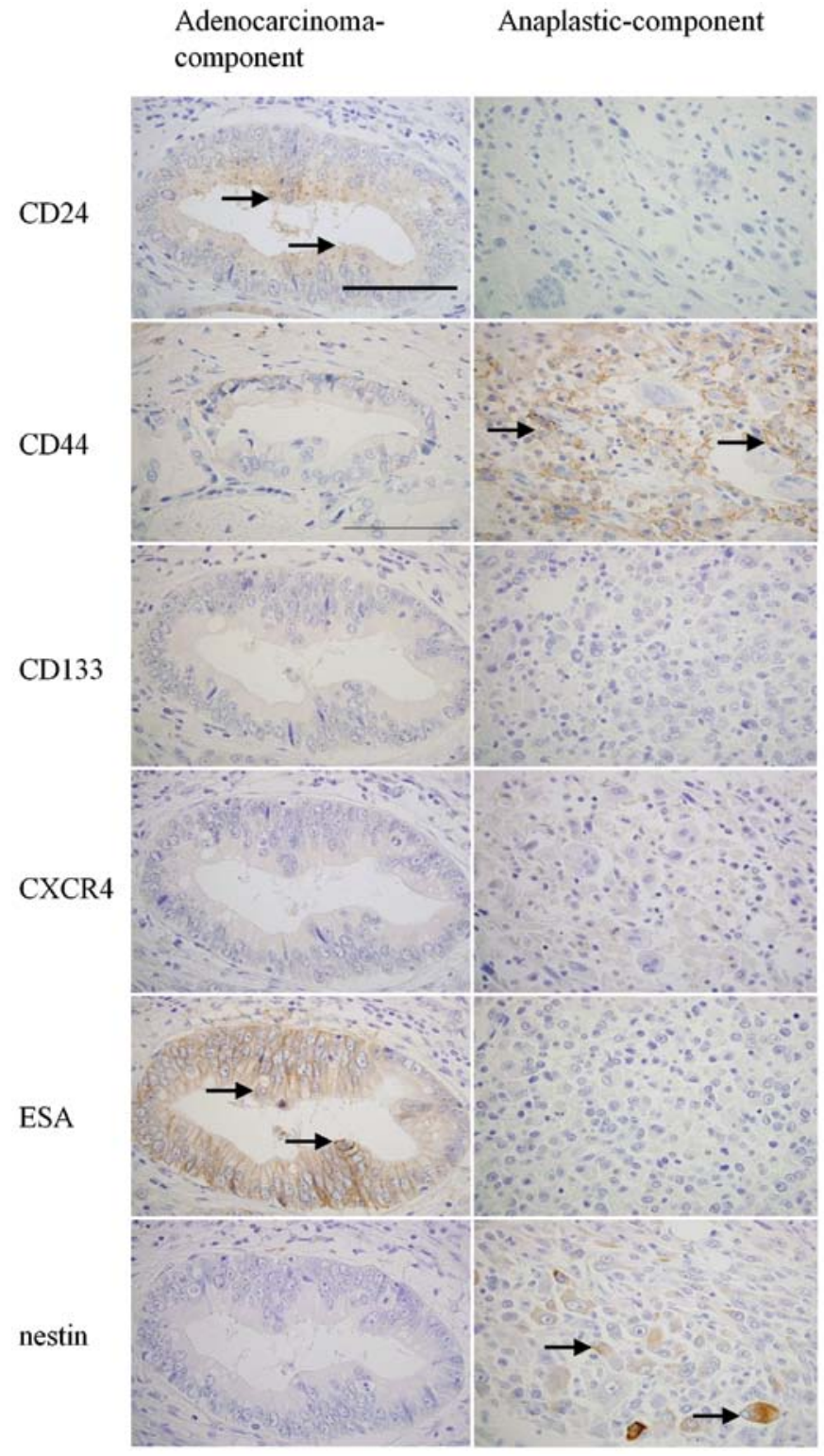

Figure 9. Immunohistochemical staining of CSC markers in anaplastic carcinoma. The arrows indicate positively stained areas. Each adenocarcinoma component and anaplastic component is from an individual case. Bar, $100 \mu \mathrm{m}$.

ical variety of PDACs determines the CSC marker expression levels. Therefore, we analyzed two PDAC subtypes, adenosquamous carcinoma $(\mathrm{N}=7)$ and anaplastic carcinoma $(\mathrm{N}=1)$, which consist of histologically different cancer cells within an individual sample. In adenosquamous carcinoma, CD133 and ESA were expressed predominantly in the adenocarcinoma component, whereas CD44 was far more strongly expressed in the squamous cell carcinoma component (Fig. 8). CD24, CXCR4, and nestin were expressed at similar levels in each component. ESA showed significantly higher expression in the adenocarcinoma component than in the squamous cell carcinoma component (Table IV, $\mathrm{P}=0.0048$ ). In anaplastic carcinoma, CD24 and ESA were predominantly expressed in the adenocarcinoma component, while CD44 and nestin were predominantly expressed in the anaplastic component (Fig. 9). These results indicate that expression of each CSC 
Table IV. Expression of stem cell markers in each component of adenosquamous carcinomas.

\begin{tabular}{|c|c|c|c|c|c|c|}
\hline & $\mathrm{CD} 24$ & $\mathrm{CD} 44$ & ESA & CD133 & CXCR4 & Nestin \\
\hline Adenocarcinoma component & $7.9 \pm 2.1$ & $12.9 \pm 4.1$ & $38.6 \pm 7.3^{\mathrm{a}}$ & $17.1 \pm 6.1$ & $16.4 \pm 4.9$ & $3.2 \pm 1.2$ \\
\hline Squamous cell carcinoma component & $5.0 \pm 1.6$ & $22.5 \pm 4.4$ & $23.6 \pm 5.4$ & $3.9 \pm 1.8$ & $13.2 \pm 5.2$ & $3.2 \pm 0.9$ \\
\hline
\end{tabular}

Data represent mean percentage of each CSC marker-positive area \pm SE. ${ }^{a} \mathrm{P}<0.05$ vs. squamous cell carcinoma component.

marker widely varies in human tissues depending on histological type.

\section{Discussion}

Identification of pancreatic CSCs is important for developing new therapies and elucidating the putative origin cell of PDAC (4). The molecules that were analyzed in the present study, have been proven to exhibit pancreatic CSC-specific expression in previous in vitro and/or in vivo studies. The biological roles of each CSC marker are widely different. CD24 and CD44 function as adhesion molecules $(19,20)$; CD133 is related to cell polarity, which is required for cell movement and asymmetric cell division (21); CXCR4 functions as a chemokine receptor (10); ESA is considered to be a morphoregulatory molecule (22), and nestin, a class VI intermediate filament proteins, is a marker of exocrine progenitors of normal pancreatic tissues and involved in cell migration and cell cycle $(18,23,24)$.

In the present study, the expression level of each CSC marker, except for CD133, increased with increasing progression through the PanIN-to-PDAC sequence. ESA showed significantly higher expression starting from PanIN-1, CD44 and CXCR4 from PanIN-2, CD24 from PanIN-3, and nestin from PDAC (not in PanINs). We also confirmed that proliferative activity increased according to the progression of the PanIN-to-PDAC sequence in the studied tissue samples. These results indicate that each CSC marker is expressed at different stages of PDAC carcinogenesis, and that the expressions of CSC markers may relate to the proliferative activity of PanIN and PDAC.

Some of the CSC markers were related to venous invasion or histological grade; CD133 was positively correlated with venous invasion, and CXCR4 and ESA were correlated with well-differentiated PDAC. These data indicate that these markers may be essential for metastasis or differentiation of PDACs, as previously reported (10,25). Our analyses of adenosquamous carcinoma and anaplastic carcinoma cases clearly showed different expression patterns of CSC markers in each cellular component.

In conclusion, our findings show that CSC marker expressions are related to carcinogenesis via the PanIN-to-PDAC sequence. Furthermore, CSC markers may contribute to proliferation, differentiation, invasiveness, or histological types of PDAC. Our present results did not indicate any single marker as being most important and specific for PDAC. A major CSC marker can vary depending on various features of cancer cases; consequently, analysis of expression level and localization of a CSC marker in each step of PDAC progression may prove useful for developing new detection and therapeutic modalities for PDAC.

\section{Acknowledgments}

The authors thank Ms. Y. Kawamoto, Ms. T. Suzuki, Ms. K. Kawahara and Mr. Y. Yanagisawa (Departments of Pathology and Integrative Oncological Pathology) for their excellent technical assistance. We express our appreciation to Dr Shin-ichi Tsuchiya (Division of Surgical Pathology, Nippon Medical School Hospital) for preparing tissue blocks. This study was supported by a Grant-in-Aid for Young Scientists (A, no. 22689038 to Y.M.), a Grant-in-Aid for Challenging Exploratory Research (No. 23650604 to Y.M.), and a Grantin-Aid for Scientific Research (C, no.22591531 to T.I.) from the Japan Society for the Promotion of Science.

\section{References}

1. Bissell MJ and Labarge MA: Context, tissue plasticity, and cancer: are tumor stem cells also regulated by the microenvironment? Cancer Cell 7: 17-23, 2005.

2. Reya T, Morrison SJ, Clarke MF and Weissman IL: Stem cells, cancer, and cancer stem cells. Nature 414: 105-111, 2001.

3. Jemal A, Siegel R, Ward E, et al: Cancer statistics, 2008. CA Cancer J Clin 58: 71-96, 2008.

4. Hruban RH, Maitra A and Goggins M: Update on pancreatic intraepithelial neoplasia. Int J Clin Exp Pathol 1: 306-316, 2008.

5. Li C, Heidt DG, Dalerba P, et al: Identification of pancreatic cancer stem cells. Cancer Res 67: 1030-1037, 2007.

6. Huang P, Wang CY, Gou SM, Wu HS, Liu T and Xiong JX: Isolation and biological analysis of tumor stem cells from pancreatic adenocarcinoma. World J Gastroenterol 14: 3903-3907, 2008.

7. Ikenaga N, Ohuchida K, Mizumoto K, et al: Characterization of CD24 expression in intraductal papillary mucinous neoplasms and ductal carcinoma of the pancreas. Hum Pathol 41: 1466-1474, 2010.

8. Hong SP, Wen J, Bang S, Park S and Song SY: CD44-positive cells are responsible for gemcitabine resistance in pancreatic cancer cells. Int J Cancer 125: 2323-2331, 2009.

9. Li C, Wu JJ, Hynes M, et al: c-Met is a marker of pancreatic cancer stem cells and therapeutic target. Gastroenterology 141: 2218-2227.e5, 2011.

10. Hermann PC, Huber SL, Herrler T, et al: Distinct populations of cancer stem cells determine tumor growth and metastatic activity in human pancreatic cancer. Cell Stem Cell 1: 313-323, 2007.

11. Olempska M, Eisenach PA, Ammerpohl O, Ungefroren H, Fandrich $\mathrm{F}$ and Kalthoff $\mathrm{H}$ : Detection of tumor stem cell markers in pancreatic carcinoma cell lines. Hepatobiliary Pancreat Dis Int 6: 92-97, 2007.

12. Kim MP, Fleming JB, Wang H, et al: ALDH activity selectively defines an enhanced tumor-initiating cell population relative to CD133 expression in human pancreatic adenocarcinoma. PLoS One 6: e20636, 2011.

13. Maeda S, Shinchi H, Kurahara H, et al: CD133 expression is correlated with lymph node metastasis and vascular endothelial growth factor-C expression in pancreatic cancer. $\mathrm{Br} \mathrm{J}$ Cancer 98: 1389-1397, 2008 
14. Marechal R, Demetter P, Nagy N, et al: High expression of CXCR4 may predict poor survival in resected pancreatic adenocarcinoma. Br J Cancer 100: 1444-1451, 2009.

15. Kawamoto M, Ishiwata T, Cho K, et al: Nestin expression correlates with nerve and retroperitoneal tissue invasion in pancreatic cancer. Hum Pathol 40: 189-198, 2009.

16. Clevers H: The cancer stem cell: premises, promises and challenges. Nat Med 17: 313-319, 2011

17. Hruban RH, Adsay NV, Albores-Saavedra J, et al: Pancreatic intraepithelial neoplasia: a new nomenclature and classification system for pancreatic duct lesions. Am J Surg Pathol 25: 579-586, 2001.

18. Carriere C, Seeley ES, Goetze T, Longnecker DS and Korc M: The Nestin progenitor lineage is the compartment of origin for pancreatic intraepithelial neoplasia. Proc Natl Acad Sci USA 104: 4437-4442, 2007.

19. Aigner S, Sthoeger ZM, Fogel M, et al: CD24, a mucin-type glycoprotein, is a ligand for P-selectin on human tumor cells. Blood 89: 3385-3395, 1997.

20. Ponta H, Sherman L and Herrlich PA: CD44: from adhesion molecules to signalling regulators. Nat Rev Mol Cell Biol 4: 33-45, 2003.
21. Immervoll H, Hoem D, Sakariassen PO, Steffensen OJ and Molven A: Expression of the 'stem cell marker' CD133 in pancreas and pancreatic ductal adenocarcinomas. BMC Cancer 8: 48,2008

22. Raffel A, Eisenberger CF, Cupisti K, et al: Increased EpCAM expression in malignant insulinoma: potential clinical implications. Eur J Endocrinol 162: 391-398, 2010.

23. Ishiwata T, Matsuda $Y$ and Naito Z: Nestin in gastrointestinal and other cancers: effects on cells and tumor angiogenesis. World J Gastroenterol 17: 409-418, 2011.

24. Matsuda Y, Naito Z, Kawahara K, Nakazawa N, Korc M and Ishiwata T: Nestin is a novel target for suppressing pancreatic cancer cell migration, invasion and metastasis. Cancer Biol Ther 11: 512-523, 2011.

25. Lee HJ, You DD, Choi DW, et al: Significance of CD133 as a cancer stem cell markers focusing on the tumorigenicity of pancreatic cancer cell lines. J Korean Surg Soc 81: 263-270, 2011. 